\begin{tabular}{|l|l|l|}
\hline & $\begin{array}{l}\text { Principales dates } \\
\text { Novembre } 1638 \text { - Naissance à Drumoak (Écosse) }\end{array}$ \\
\hline 1663 & Parution de Optica Promota \\
\hline 1667 & Parution de Vera circuli et hyperbolae quadratura \\
\hline 1668 & $\begin{array}{l}\text { Elu fellow de la Royal Society } \\
\text { Titulaire de la Regius Chair of mathematics à Saint-Andrews }\end{array}$ \\
\hline 1673 & Hooke construit le premier télescope grégorien \\
\hline 1674 & Titulaire de la Chair of mathematics à Édimbourg \\
\hline
\end{tabular}

\title{
James Gregory
}

\section{Riad Haidar}

haidar@onera.fr

Astronome et brillant mathématicien écossais, à qui l'on doit notamment le développement en série infinie de plusieurs fonctions trigonométriques, James Gregory est surtout connu pour son invention du premier télescope catadioptrique, qui porte aujourd'hui son nom.

J ames Gregory (également orthographié Gregorie) naît en novembre 1638 à Drumoak, un village minuscule situé le long du fleuve Dee, au sud-ouest d'Aberdeen, en Ecosse. II vient au monde dans une famille auréolée de gloire. Le grand-père maternel, David Anderson, s'est forgé à Aberdeen une légende de savant et ingénieur exceptionnel. Puis son fils Alexander a été élève du célèbre algébriste François Viète [1540 - 1603], et enseigne les mathématiques à Paris. Enfin sa fille Janet, férue de géométrie, a épousé le Révérend Anglican John Gregory, formé en théologie à I'université Saint-Andrews, prêtre de la paroisse de Drumoak ; il est remarquable que cette union donnera une succession ininterrompue de scientifiques qui marqueront 200 ans d'histoire écossaise, en mathématiques, médecine, chimie...

\section{Des talents précoces}

James est le benjamin de Janet et du Révérend John. Enfant de constitution fragile, son éducation se fait d'abord à la maison. II apprend ainsi la géométrie avec sa mère puis, sous la férule de son frère David, il découvre les Éléments d'Euclide. Son aisance à jongler avec les concepts abstraits est telle qu'on l'inscrit à la Grammar School en 1651, où il révèle des dons rares. II intègre ensuite le Marischal College de l'université d'Aberdeen, d'où il sort diplômé en 1657. II n'a pas encore 19 ans.

Inspiré par les travaux de Kepler, et fortement encouragé par son frère David, il s'intéresse tout de suite à l'optique. Il achève en 1662 son premier livre, Optica Promota (littéralement, Progrès en Optique), où i étudie la réfraction et la réflexion de la lumière, discute de considérations astronomiques et, surtout, propose un concept révolutionnaire de télescope catadioptrique : composé de deux miroirs concaves (l'un parabolique, I'autre elliptique), le télescope grégorien s'affranchit de certaines aberrations des dispositifs purement réfractifs et permet, en repliant le trajet optique, un gain considérable sur la longueur du tube.

Toutefois, la fabrication des miroirs pose des problèmes que les techniques de l'époque peinent à surmonter. En 1663 Gregory se rend à Londres, où son livre lui ouvre les portes des milieux scientifiques. II y fait la connaissance du célèbre Robert Moray [1609 - 1673], compatriote écossais et fondateur de la Royal Society, à qui il fait une forte impression; et surtout du mathématicien John Collins [1625 - 1683], avec il se lie d'une amitié qui durera toute leur vie et qui le recommande auprès des meilleurs artisans. Mais aucune de ses tentatives 
pour fabriquer son télescope n'aboutit... II faudra attendre dix ans pour que le génie expérimental de Robert Hooke [1635 - 1703] parvienne à concrétiser les calculs théoriques de Gregory. Le télescope grégorien s'impose alors comme l'outil classique de l'astronome du $18^{\text {e }}$ siècle, supplantant notamment son rival newtonien.

\section{Voyage en Europe}

En 1664 Gregory se rend sur le Continent. Après un passage par les Flandres, Paris et Rome, il s'installe en République de Venise, à l'université de Padoue, où il intègre l'équipe du mathématicien Stefano degli Angeli [1623 - 1697]. II s'initie pendant quatre années aux travaux de Cavalieri [1598 - 1647] sur les développements en série et leur application à la géométrie. Dans la Sérénissime, malgré l'atmosphère oppressante de I'inquisition italienne, il s'épanouit intellectuellement. La puissance et la modernité des nouveaux outils mathématiques l'éblouissent et stimulent sa créativité. II publie Vera circuli et hyperbolae quadratura en 1667 et Geometriae pars universalis en 1668, dans lesquels il donne les développements en série de sinus, cosinus, arc tangente... II établit, pour la première fois, que la méthode des tangentes (la différentiation) est l'opération inverse de celle des quadratures (l'intégration). II déduit également l'expression du nombre $\pi$ et a l'intuition de sa transcendance, mais sans parvenir à la prouver parfaitement.

Ses livres présentent une remarquable collection de concepts originaux, et lui forgent une solide réputation de penseur. Ils lui valent ainsi une nomination immédiate comme fellow de la jeune Royal Society mais aussi, dans le même temps, l'inimitié de Christiaan Huygens [1629 - 1695] à qui il a envoyé, comme un hommage, un exemplaire de son manuscrit. La réaction du maître Flamand est inouie : il réagit puissamment par la publication, quelques mois plus tard, d'une liste de commentaires où il démolit, ici, l'argumentaire de Gregory et revendique, là, la paternité de certains résultats. Un œil averti comprend immédiatement qu'Huygens a manqué d'équité envers son cadet, et que Gregory a obtenu ses résultats en toute indépendance, mais il n'empêche : I'attaque vexe la fierté de Gregory. II réplique par un nouvel ouvrage, Exercitationes Geometricae, qu'il construit comme un défi à Huygens (" I let the reader who has compared this work with others judge what is mine and what belongs to another $»$. Mais, pour aller au bout de la logique du défi et surtout prévenir toute accusation de plagiat, il dissimule les éléments clefs de son raisonnement. Pire : rendu frileux, dans cette atmosphère si particulière d'une Europe qui émerge lentement du Moyen Âge et craint de s'émanciper de l'influence des Anciens, il hésite désormais à publier, de sorte qu'une large part de ses travaux resteront inconnus jusqu'au milieu du $20^{\circ}$ siècle... le privant de la reconnaissance que ses découvertes auraient dû lui valoir. C'est le cas, notamment, du développement limité qu'il invente plus de 40 ans avant sa publication par Brook Taylor [1685 - 1731].

\section{Astronomie et grandes formules}

II retourne en Angleterre en 1668, et séjourne quelques mois à Londres chez John Collins, dont l'amicale sollicitude est un baume revigorant, et qui l'informe des derniers développements mathématiques. Durant l'été, son ami et protecteur Robert Moray use de son influence auprès de Charles II pour créer la première Regius Chair of Mathematics de l'université Saint-Andrews, un poste hors-cadre taillé sur mesure pour permettre à Gregory de poursuivre librement ses recherches en mathématiques.

Gregory arrive ainsi, à l'automne 1868, dans une université bicentenaire qu'il découvre enfermée dans une pesante tradition médiévale, et superbement ignorante des derniers développements scientifiques. Cette même année, il retrouve son amie d'enfance Mary, la fille du peintre portraitiste George Jamesone, qu'il épouse en 1669. Le couple aura deux filles et un garçon. Mary est une femme cultivée, qui lui apporte une stabilité familiale et un soutien intellectuel inébranlable. Aussi, bien qu'isolé dans sa province, Gregory maintient une activité fertile. II conserve un contact étroit avec le milieu scientifique à travers une correspondance nourrie avec son ami londonien Collins, et mène ses meilleurs travaux mathématiques - malheureusement non publiés, mais dont les archives nous sont parvenues. Observateur éclairé, il découvre le principe du réseau de diffraction en remarquant l'irisation de la lumière filtrée par une plume d'oiseau marin.

En 1673, après de longues discussions, l'université autorise enfin Gregory à entreprendre la construction d'un observatoire, mais il lui revient de réunir les fonds. Pugnace et plein de ressources, il organise des collectes à la sortie des églises, s'informe auprès de John Flamsteed qui est Astronome Royal... Malheureusement, ostracisé par les professeurs de l'université dont l'enseignement dogmatique se heurte aux concepts modernes qu'il présente aux étudiants, Gregory doit quitter Saint-Andrews. Il est accueilli à Édimbourg en 1674, à la chaire de mathématiques. Mais peu de temps après, une nuit d'octobre 1675, après une séance d'astronomie où il montre les satellites de Jupiter à quelques étudiants, il est atteint d'une attaque cérébrale et perd la vue. II décède au bout de quelques jours, à l'âge de 36 ans, laissant derrière lui une œuvre considérable, et notamment ses Universal methods in geometry and analysis que les historiens exhumeront 250 ans plus tard.

\section{Références}

[1] H.W. Tunrbull, The Tercentenary of the birth of J. Gregory (1938).

[2] J.J. O'Connor and E.F. Robertson, Biography of J. Gregory, University of St Andrews (2000).

[3] A. Malet, Studies on J. Gregory, Princeton University Ph.D. thesis (1989). 